\title{
A NEW TRAPEZIUM SYSTEM ASSOCIATED WITH A COMPACT H II REGION
}

\author{
S. Sharpless, J.L. Pipher, M.P. Savedoff \\ and S. SchurmanN
}

C.E. Kenneth Mees Observatory

University of Rochester

The region $\mathrm{S} 106=\mathrm{G} 76.4-0.6$ has been photographed in the $8000 \AA$ region with the Carnegie image converter attached to the $0.6 \mathrm{~m}$ telescope at the Mees Observatory. This was done in connection with a larger program designed to assemble radio, infrared, and optical data on compact HII regions.

S106 has angular dimensions of $1^{\prime}$ by $3^{\prime}$ and has relatively high surface brightness. Its kinematic distance based on $\mathrm{H} 109 \alpha$ observations (Reinfenstein, $e t$ al. 1970 ) is $2.3 \mathrm{kpc} \pm 3.0$.

Several bright maxima are observed within the nebula at wavelengths of $12.6 \mu$ and $2.7 \mathrm{GHz}$. These are believed to be the result of thermal radiation from hot dust and free-free emission from ionized gas, respectively. At the nominal distance their linear sizes are of the order of $0.2 \mathrm{pc}$ and they may be similar to the "cocoon" type objects discussed by various authors.

Another bright maximum is visible at $3.5 \mu$. Its spectrum in the $2-20 \mu$ range displays a marked hump suggestive of a hot source which is highly reddened by dust associated with the nebula. An image converter photograph having an effective wavelength of about $8000 \AA$ shows an object at the same location as the $3.5 \mu$ source. The positional agreement is within 2 " which is the resolution of the photograph. An infrared source at approximately this position has already been suggested by Sibille et al. (1975). We believe that there is a strong possibility that this object is the exciting star of S106. The suggestion that it is highly reddened is confirmed by the fact that is does not appear on image converter photographs taken at shorter wavelengths. The object does not appear as a single star on the infrared photograph but rather as a multiple star having angular dimensions of approximately $5^{\prime \prime}$. It is interesiing to note that the Orion Trapezium, $\theta^{1}$ Orionis, would appear to have this angular size if viewed from a distance equal to that of S106. We believe, therefore, that we have found a very highly reddened, trapezium system. This object appears near apparent infrared magnitude of about 20 . If we assume its absolute magnitude to be similar to that of $\theta^{1}$ Orionis, and its distance to be $2.3 \mathrm{kpc}$, then the resulting infrared absorption is in the neighborhood of $14 \mathrm{mag}$. The corresponding visual absorption is 27 mag. Most of this absorption must be assumed to take place within, or immediately surrounding, the HII region. It is believed that a large dust component associated with an HII region is a sign of extreme youth (Terzian and Balick 1974). We may therefore be observing in S106 an exceptionally young trapezium system.

\section{REFERENCES}

Reifenstein III, E. C., Wilson, T. L., and Burke, B. F. 1970, Astron. and Astrophys., 4, 357.

Sibille, F., Bergeat, J., Lunel, M., and Kandel, R. 1975, Astron. and Astrophys., 40, 441.

Terzian, Y., and Balick, B. 1974, Fund. of Cosmic Physics, $\mathbf{1}, 301$.

\section{DISCUSSION}

Poveda: I find it very exciting that your new trapezium fits into an evolution sequence where the Kleinmann-Low Nebula could represent the very earliest stage of development; next comes your trapezium, and finally, when the cocoon nebula dissipates, the Orion trapezium. 